\title{
Intraoperative Neurovascular Bundle Preservation With Hyaluronic Acid During Radical Brachytherapy for Localized Prostate Cancer: Technique
}

Pedro J. Prada ( $\sim$ pprada@telecable.es )

Hospital Universitario Marques de Valdecilla https://orcid.org/0000-0002-7378-7591

\section{J. Cardenal}

Hospital Universitario Marques de Valdecilla

\section{A. García Blanco}

Hospital Universitario Marques de Valdecilla

M. Ferri

Hospital Universitario Marques de Valdecilla

E. Arrojo

Hospital Universitario Marques de Valdecilla

J. Anchuelo

Hospital Universitario Marques de Valdecilla

\section{Diaz de Cerio}

Hospital Universitario Marques de Valdecilla

\section{N. Sierrasesumaga}

Hospital Universitario Marques de Valdecilla

\section{P. Lastra}

Hospital Universitario Marques de Valdecilla

\section{A. Fernández}

Hospital Universitario Marques de Valdecilla

\section{A. Vázquez}

Hospital Universitario Marques de Valdecilla

\section{S. Ruiz Arrebola}

Hospital Universitario Marques de Valdecilla

\section{Research article}

Keywords: Brachytherapy, Neurovascular Bundle, Preservation, Technique.

Posted Date: October 5th, 2020

DOl: https://doi.org/10.21203/rs.3.rs-58759/v1 
License: (c) (i) This work is licensed under a Creative Commons Attribution 4.0 International License. Read Full License 


\section{Abstract}

Background: To evaluate the reduction, the absorbed dose delivered to the neurovascular bundle (NB) in patients with localized prostate cancer treated with only HDR brachytherapy and neurovascular bundle protection with Hyaluronic acid $(\mathrm{HA})$ on the side of the prostate to increase the distance from NB to the radioactive sources.

Material and Methods: This is the first published report in the medical literature that study a new approach to decrease neurovascular bundle toxicity and improve quality of life for patients with prostate cancer treated with radical brachytherapy as monotherapy. Transperineal HA injection on the side of the prostate into the lateral aspect of the prostate fat was used to consistently displace several autonomic fibres and vessels the lateral wall of the prostate away from radiation sources.

Results: When a protection in the form of a HA layer is placed, the reduction effect at the maximum dose is between $46 \%$ and $54 \%$ (calculated values), which means the method for protection is highly recommended. The values of the absorbed dose calculated in this project have been compared with the ones given by the treatment planning system. These calculated values are similar than the ones given by the treatment planning system $(56 \pm 2)$.

Conclusions: This newly created space decreases absorbed dose in the neurovascular bundle, calculated with the TPS and measuremed by microMOSFET due to the thickness of HA.

\section{Background}

A variety of brachytherapy modalities are available for the radical treatment of localized prostate cancer. Low-dose rate (LDR) and High-dose-rate (HDR) brachytherapy are accepted, both treatments achieving similar rates of biochemical control although with differing types of morbidities [1-2].

Radical brachytherapy is an accepted, effective and safe therapy for localized prostate cancer. Contemporary series examining brachytherapy (BT), indicate excellent cancer-specific outcomes among younger patients [3-5]. Brachytherapy is equally efficient than other interventions (prostatectomy, external beam radiotherapy) but less harmful and is especially attractive for patients with early prostate cancer [6-9].

The associated morbidity to the brachytherapy treatment has decreased the last decade as result of improvement in the brachytherapy technique. Young men with longer life expectancies, are the population most likely to benefit from reduced normal tissue toxicity, particularly erectile preservation.

The purpose of the present study, was to determine the reduction the absorbed dose delivered to the neurovascular bundle (NB) using microMOSFET detectors ("in vivo" dosimetry) in patients with localized prostate cancer treated with only HDR brachytherapy and neurovascular bundle protection with Hyaluronic acid $(\mathrm{HA})$ on the side of the prostate to increase the distance from NB to the radioactive 
sources. With this technique, we believe much of the side effects can be either reduced or even eliminated.

\section{Material And Methods}

This is the first published report in the medical literature that study a new approach to decrease neurovascular bundle toxicity and improve quality of life for patients with prostate cancer treated with radical brachytherapy as monotherapy. Transperineal HA injection on the side of the prostate into the lateral aspect of the prostate fat was used to consistently displace several autonomic fibres (afferent and efferent) and vessels the lateral wall of the prostate away from the effective and biologic life of radiation sources. In low and low-intermediate risk patients treated with HDR monotherapy, the HA injection was administered before dose delivery for HDR patients. This study was approved by the institutional ethics committee and all patients signed informed consent.

\section{Sexual function}

The sympathetic and parasympathetic nervous system both play an important role in sexual function. Sacral parasympathetic fibres from S2-4 travelling through the pelvic plexus and forming the nervi erigentes dorsolateral to the bladder and prostate are responsible for the blood flow into the corpora cavernosa resulting in penile erection. Sympathetic fibres are responsible for emission of semen from the seminal vesicles into the prostatic urethra and antegrade ejaculation. Thus, iatrogenic damage to the parasympathetic and sympathetic pathways during radical brachytherapy may result in erectile and ejaculation dysfunction $[10,11]$.

\section{Study model}

The injection of $\mathrm{HA}$ is performed before needle placement creating a minimum of a $1.5-2 \mathrm{~cm}$ space between the prostate capsule and neurovascular bundle. Having finished the HDR brachytherapy needle placement and before dose delivery, microMOSFET detectors are placed within the prostatic capsule and in neurovascular bundle. Intraoperative trans-rectal ultrasound (TRUS) is used for verification of the microMOSFET placement. In this fashion, we measure the delivered dose to both critical organs during the treatment "in vivo".

Magnetic Resonance pre and post-implant imaging are used to assess the dimensions of the new space create.

The patient received one implant and one fraction of HDR. Fraction dose was $22 \mathrm{~Gy}$.

Brachytherapy procedures were done under spinal anesthesia. The needles were positioned by transperineal placement under real time TRUS guidance using a template. Axial cross-sections were captured in $5 \mathrm{~mm}$ steps and transferred to the Treatment Planning Software. Prostate gland, normal structures (urethra and rectum) and needle positions were identified and mapped based on the 
ultrasound image. Dose optimization was done on the reconstructed applicator geometry using dose point and manual optimization algorithms to determine dwell positions and times.

The prostate without safety margins was then defined as the planning target volume (PTV) to be treated with the prescribed dose (PD). Based on the dose volume histograms (DVH) data, the quality of plans and implants was evaluated using following indicators:

- The rectal dose was calculated at the anterior edge of the TRUS probe and was limited to $\leq 75 \%$ of the prescription dose.

- The dose to any segment of the urethra was limited to $\leq 110 \%$ of the prescription dose. V120 and D100 of the prostatic urethra were determined (volume that received a dose of $120 \%$ and dose delivered to $100 \%$ of the urethra).

- The PTV V90, V100, V150 and V200 (\% of PTV receiving $90 \%, 100 \%, 150 \%$ and $200 \%$ of the PD) were recorded.

- D90 (dose delivered to $90 \%$ of the PTV) was calculated.

All patients are discharged from the center on the same day of the procedure between 6-8 hours of implantation.

\section{Technique of hyaluronic acid injection}

The injection of HA is performed before needle placement. Based on our experience of rectal protection with HA in prostate cancer Brachytherapy $[12,13]$. Below we describe the technique.

Step 1: The TRUS probe with the transperineal template is placed and fixed in the standard fashion. Magnetic Resonance pre-implant imaging are fused with the intraoperative TRUS image. Then all treatment needles are placed under TRUS guidance.

Step 2: Using TRUS guidance, an additional needle is placed guiding the needle tip into the peri-prostatic fat, between the lateral prostate capsule (treated volume) and the neurovascular bundle, at the level of the maximum transverse diameter of the prostate (reference level). Under direct TRUS guidance, the needle tip is advanced to the level of the seminal vesicles and then to the level of the prostatic apex. Extreme care is taken not to perforate the prostate capsule with the needle tip.

Step 3: The additional needle is connected to the syringe containing HA. After aspirating to ensure that contact with a vessel had not occurred, between 6 and $10 \mathrm{cc}$ of HA is injected within lateral prostate capsule and the neurovascular bundle. This is performed under continuous TRUS guidance to view and verify the new space created by the injection. The total injected amount is related to the need for systematically creating a minimum of a $1.5-\mathrm{cm}$ space between the prostate, seminal vesicles, apex and neurovascular bundle throughout this length. 
Step 4: Under TRUS guidance, the neurovascular and lateral prostatic catheters with the microMOSFET are placed. The absorbed dose is calculated as the difference of the dose values between the points separated by the HA. We calculated the value of this decrease using the Oncentra Prostate planning system of Nucletron, and measuremed this value using TN-502-RDM MOSFET detectors, hereafter microMOSFETs, manufactured by Best Medical Canada (Ottawa, Canada).

The dimensions of microMOSFET detectors enabling their insertion in needles used for HDR brachytherapy. Detector response in voltage is measured before and immediately after each exposure or treatment completed. The voltage difference between these measurements is proportional to the absorbed dose, which is obtained by applying a pre-determined calibration factor (CF) in Gy/V. In the clinical setting, these detectors are inserted in additional needles to those used in the treatment, placed at both ends of the HA thickness, allowing measurement of the true dose delivered. After correct insertion of the tip of the needle, monitored by US, the detector is placed in the end. In these conditions, the position of the sensitive part of the microMOSFET is approximately to $8 \mathrm{~mm}$ from the tip of the needle. The measurement system includes a mobile MOSFET Reader TN-RD-16 electrometer that can simultaneously measure up to five detectors.

In the same plane of the ultrasound image in which the two microMOSFET are placed, and in the two points marked by the needles in which the detectors are for each patient, the absorbed dose is calculated with the Treatment planning system (TPS) once the planning of the treatment has completed. In this way we can compare the value of the decrease absorbed dose obtained by both the TPS and the microMOSFET.

The microMOSFET was calibrated employed a water phantom. The absorbed dose for calibration at the measurement was $1 \mathrm{~Gy}$, the source-detector calibration distance was $3 \mathrm{~cm}$, and the dwell time required for this absorbed dose value at the calibration point was determined using the Oncentra Prostate planning system. The CF for twenty microMOSFET detector was obtained, taking five consecutive measurements for each, to calculate its reproducibility.

All measurements and treatments were performed using Flexitron afterloader and the ${ }^{192}$ Ir V2r source (Nucletron/Elekta, Stockholm, Sweden).

Step 5: After the HDR treatment is completed, both catheters containing the microMOSFET detectors are removed for reading.

\section{Uncertainties}

We considered two groups of uncertainties, those associated with dose calculation by the TPS and those related to dose measure by microMOSFET detector $[14,15]$. For uncertainties of calculated absorbed dose, we considered source intensity (Air kerma strength, $S_{K}$ ) and the interpolation associated with the TPS. For uncertainties of measured absorbed dose, uncertainty related to the global CF corresponding to 
each detector, we consider in addition to the previous uncertainties, the source-detector calibration distance, the electrometer resolution and the reproducibility.

\section{Hyaluronic acid}

The HA is a polysaccharide normally found in human tissues as a component of the connective tissue and it has an absorption coefficient equal to water. Normally, it plays a vital role on the skin and in the synovial fluid of the joints. It is normally degradable by the normal enzymatic system in relative short time. However, to make it last for months when used for the treatment of skin wrinkles and osteoarthritis, the compound is modified making it stable for a duration close to 1 year before it is reabsorbed by the body $[16,17]$. Only one type of HA is used in this trial.

\section{Results}

This is the first report in the medical literature using HA as a neurovascular bundle protector in patients with prostate cancer treated with only HDR interstitial brachytherapy as monotherapy in one fraction of $22 \mathrm{~Gy}$.

\section{Patient characteristics}

Our decision for neurovascular bundle preservation was considered appropriate in young men at the time of diagnosis and more interested in preserving sexual function, when biopsy examination demonstrated and confirmed imaging findings of organ-confined cancer with no suggestion of extracapsular extension to the neurovascular bundle. The biopsies and imaging's were also evaluated by experienced pathologists and radiologists.

\section{Radiologic studies}

Figures 1 and 2 corresponds to a magnetic resonance image (MRI) before and after HA injection demonstrating the newly created space between the prostate capsule and the neurovascular bundle.

\section{microMOSFET Analysis}

The mean values of the decrease in absorbed doses due to the HA thickness for three patients is exhibits in Table 1. TPS dose calculation and microMOSFET detector dose measurement are expressed in Gy and percentage. HA thicknesses were measured in the ultrasound image and are expressed in $\mathrm{mm}$. All uncertainties correspond to a coverage factor $k=1$.

The values in percent of the uncertainties that contribute to the TPS dose calculation are for $S_{k} 1.5 \%$ and TPS interpolation $2.6 \%$, both taken from [18]. We estimated the uncertainty related to dose calculation as the mean quadratic root of the two previous uncertainties considered, that is $3.0 \%$. We followed the same procedure to calculate the uncertainty of measurement of the absorbed dose, related to the global detector CF, that is $4.3 \%$, but also considering other sources of uncertainty such as source-microMOSFET 
distance, $2.0 \%$, resolution of electrometer, $0.009 \%$, and reproducibility measuremed for twenty detectors, $2.3 \%$. We considered an imprecision in the detector position of $0.5 \mathrm{~mm}$ to calculate the uncertainty in the calibration associated with source-detector distance. Considering the relative standard deviation (RSD) of five measurements for each detector, the reproducibility was calculated as the mean relative standard deviation for twenty detectors. RSD is defined as the ratio of the standard deviation $(\sigma)$ to the absolute value of mean $(|\mu|)$. The uncertainty HA thicknesses are given by the precision of the measurement software of the ultrasound system. All uncertainties of the mean measurements for a coverage factor $\mathrm{k}=$ 1.

\section{Tolerance}

We have not seen side effects related to the injection or the compound itself. There is no toxicity in the fat tissue neurovascular bundle or in rectal function. Patients have not complained of pain, tenesmus, pelvis pressure, or sensation of rectal filling. During the posttreatment follow-up evaluation, no patients complained of discomfort that could be attributed to the HA injection.

Because this work was started very recently, the results will be reported separately in a different publication after enough cases are performed for statistical analysis.

\section{Discussion}

The relationship between the probability of tumor control and dose has long been established. [19, 20]. The principal aim of conformal brachytherapy is to reduce irradiation to organs at risk, which enables a higher dose to the target volume. However, the increase in radiation dose to the prostate also has an impact of higher probability of toxicity, in the neurovascular bundle [21]. The post-radiation injury mechanism is related to the damage of the vascular endothelium the small vessels, arterioles and subsequent development of edema and fibrosis in nervous, vascular and muscular tissue [22]. The consequence is the increased fragility of the neurovascular bundle and their tendency to erectile dysfunction.

The technique developed appears to be effective in keeping the neurovascular bundle away from the ionizing radiation. Hyaluronic acid is never injected in the prostatic stroma, but placed into the periprostatic fat and is absorbed locally by the lipocytes, it does not leak into the prostatic stroma. For these reasons, we felt it was safe to use.

When a protection in the form of a HA layer is placed, the reduction effect at the maximum dose is between $46 \%$ and $54 \%$ (calculated values), which means the method for protection is highly recommended. The values of the absorbed dose calculated in this project have been compared with the ones given by the treatment planning system. These calculated values are similar than the ones given by the treatment planning system ( $56 \pm 2$ ), as can be seen in Table 1 . This may be caused by the multiple approximations we've taken into account and because of the layout of the needles. 
When the treatment is external beam radiotherapy or brachytherapy the ionizing radiation can damage the neurovascular bundle. To avoid morbidity, it is recommended to reduction of dose and minimize the volume of neurovascular bundle receiving high doses but these procedures increase the likelihood of tumor persistence, thereby compromising oncologic control.

Several authors $[23,24,25]$ will be testing the same concept to critical structures (rectum) and presented a correlation of increasing rectal mucosal dose with increased rectal toxicity. In their multivariate analysis, dose was the only significant factor associated with Grade 2-3 of rectal toxicity. They concluded that chronic rectal toxicity is sequelae of high dose conformal treatment of prostate cancer. Appropriate shielding of the rectal mucosa limiting the dose and to minimize the volume of rectum receiving high doses is required to avoid a high incidence of these complications.

In the same way our previous study [13], patients with low- and intermediate-risk prostate cancer were enrolled in a randomized clinical trial.

- First group received brachytherapy alone with $1-125$

- Second group received brachytherapy alone with I-125 and rectal protection with HA. HA in the perirectal fat, to increase the distance between the prostate and the anterior rectal wall.

Of the non-hyaluronic group (first group), 4 out of 33 patients (12\%) were suffering from intermittent rectal bleeding and in these 4 patients significant mucosal damage could be found. The total percentage of visible damage was $36 \%$ of the group. No patients with bleeding were observed in the group with rectal protection ( $\mathrm{HA}$ ) and only 2 patients presented with minimal objective mucosal damage at the endoscopy.

According to our data, the increase in distance between rectum and posterior prostatic capsule created by the perirectal injection of $\mathrm{HA}$, is enough to provide a significant radiation dose reduction and toxicity.

We think that also there a correlation between increase of dose and fragility of the neurovascular bundle and their tendency to erectile dysfunction. The increase in distance between prostatic capsule and neurovascular bundle created by injection of $\mathrm{HA}$, is enough to provide a significant radiation dose reduction from HDR brachytherapy and we believe much of the side effects can be either reduced or even eliminated. No toxicity was produced from the HA or its injection.

Longer follow-up is needed to determine if there is a benefit from these planning exercises.

We are currently conducting a new study in which to increase the number of measurements and thus be able to evaluate the results with better statistics.

\section{Conclusions}

When HA is injected (under TRUS guidance) transperineally into the lateral aspect of the prostate fat and the neurovascular bundle, creating a minimum of a $1.5-\mathrm{cm}$ space, one can demonstrate by MRI. This 
newly created space decreases absorbed dose in the neurovascular bundle, calculated with the TPS and measuremed by microMOSFET due to the thickness of HA.

Longer follow-up is necessary to demonstrate the real gain with decreased neurovascular bundle doses.

\section{Abbreviations}

BT: Brachytherapy

HDR: High dose rate

LDR: Low dose rate

TRUS: The trans-rectal ultrasound

MRI: Multiparametric magnetic resonance imaging

D90: The dose that covers $90 \%$ volume of CTV

D100 urethra: Dose delivered to $100 \%$ of the urethra

DVH: Dose volume histogram

PTV: Planning target volume

PD: Prescribed dose

V90-100-150-200: \% of PTV receiving $90 \%, 100 \%, 150 \%, 200 \%$ of the PD)

V120 urethra: Volume that received a dose of $120 \%$ of the urethra

TPS: Treatment planning system

HA: hyaluronic acid

NB: Neurovascular bundle

CF: Calibration factor

RSD: Relative standard deviation

\section{Declarations}

\section{Conflicts of interest}

No potential conflict of interest. 
AUTHORS'CONTRIBUTIONS

Pedro J. Prada M.D, Ph.D: conception and design.

Juan Cardenal M.D: analysis of data.

Ana Garcia Blanco M.D: revising references.

Javier Anchuelo M.D: acquisition of data.

Maria Ferri: acquisition of data.

Iván Diaz de Cerio M.D: alignment and drafted the manuscript.

Andrés Vázquez Ph.D: analysis of data.

Samuel Ruiz Ph.D: analysis of data.

Elisabeth Arrojo M.D: acquisition of data.

Nicolás Sierrasesumaga M.D: alignment and drafted the manuscript.

Alejandro Fernández M.D: acquisition and processing of Magnetic resonance image

Pedro Lastra M.D: acquisition and processing of Magnetic resonance image

Ethics approval and consent to participate. Not applicable.

Consent for publication. Not applicable.

Availability of data and materials. Not applicable.

Competing interests. Not applicable.

Funding. Not applicable.

Acknowledgements. Not applicable.

Authors' information (optional). Not applicable. 


\section{References}

1. Lee, RW, Moughan J., Owen J, Zelefski MJ.: The 1999 Patterns of care Study of radiotherapy in localized prostatic carcinoma. A comprehensive survey of prostate brachytherapy in the United States. Cancer 2003; 98 (9): 1987-1994.

2. Battermann JJ, Boon TA, Moerland A. Results of permanent prostate brachytherapy, 13 years of experience at a single institution. Radiotherapy and Oncology 2004; 71:23-28.

3. Jang TL, Yossepowitch O, Bianco FJ Jr, Scardino PT. Low risk prostate cancer in men under age 65: the case for definitive treatment. Urol Oncol 2007; 25:510-14 3.

4. Merrick GS, Wallner KE, Butler WM et al. Brachytherapy in men aged $<$ or $=54$ years with clinically localized prostate cancer. BJU Int. 2006;98: 324-8.

5. Pedro J. Prada M.D, Ph.D ${ }^{1}$., Juan Cardenal M.D ${ }^{1}$., Ana García Blanco M.D ${ }^{1}$., et al. long-term outcomes in patients treated with brachytherapy for prostate cancer younger than 60 years of age. Strahlentherapie und Onkologie. April 2018, 194 (4):311-317.

6. Burri RJ, Ho AY, Forsythe K, Cesaretti JA, Stone NN, Stock RG. Young men have equivalent biochemical outcomes compared with older men after treatment with brachytherapy for prostate cancer. Int J Radiat Oncol Biol Phys 2010; 77: 1315-21.

7. Khan MA, Han M, Partin AW, Epstein JI, Walsh PC. Long-term cancer control of radical prostatectomy in men younger than 50 years of age: update 2003. Urology 2003; 62: 86-9.

8. Zelefsky MJ, Marion C, Fuks Z, Leibel SA. Improved. Improved biochemical disease-free survival of men younger than 60 years with prostate cancer treated with high dose conformal external beam radiotherapy. J Uro/ 2003;170: 1828-32.

9. Yamada Y, Bhatia S, Zaider M etal. Favorable clinical outcomes of three-dimensional computeroptimized high-dose-rate prostate brachytherapy in the management of localized prostate cancer. Brachytherapy 2006; 5: 157-64.

10. Walsh PC. Anatomic radical prostatectomy: evolution of the surgical technique. J Urol 1998; 160:2418-24.

11. Thomas M. Kessler, Fiona C. Burkhard, Urs E. Studer. Nerve-sparing open radical retropubic prostatectomy.Eur Urol 2007; 51: 90-97.

12. Prada PJ, Fernandez J., Martinez A., et al. Transperineal injection of hyaluronic acid in the anterior peri-rectal fat to decease rectal toxicity from radiation delivered with intensity modulated brachytherapy or EBRT for prostate cancer patients. Int J Oncol Biol Phys 2007; 69 (1):95-102.

13. Pedro J. Prada, Herminio Gonzalez, Cosuelo Menéndez., et al. Transperineal Injection of Hyaluronic Acid in the Anterior Peri-rectal Fat to Decrease Rectal Toxicity from Radiation Delivered with Low Dose Rate Brachytherapy for Prostate Cancer Patients. Brachytherapy 2009; 8(2): 210-217.

14. DeWerd LA, Ibbott GS, Meigooni AS, Mitch MG, Rivard MJ, Stump KE, Thomadsen BR and Venselaar JLM. A dosimetric uncertainty analysis for photon-emitting brachytherapy sources: Report of AAPM 
Task Group No. 138 and GEC-ESTRO. Med Phys 201; 38:782-801.

15. Kirisits C, Rivard MJ, Baltas D, Ballester F, de Brabandere M, van der Laarse R, Niatsetski Y, Papagiannis P, Hellebust TP, Pérez-Calatayud J, Tanderup K, Venselaar LMJ and Siebert FA. Review of clinical brachytherapy uncertainties: Analysis guidelines of GEC-ESTRO and the AAPM. Radiother Oncol 2014; 110:199-212.

16. George E. Intra-articular hyaluronan treatment for osteoarthritis. Ann Rheum Dis 1998; 57:637-640.

17. Wobig M, Bach G, Beks P, et al. The role of elastoviscosity in the efficacy of viscosupplementation for osteoarthritis of the knee: A comparison of Hyalan G-F20 and a lower molecular weight hyaluronan. Clin Ther 1999; 21:1549-1562.

18. Roshau JN and Hintenlang DE. Characterization of the angular response of an isotropic MOSFET dosimeter. Health Phys 2003;84:376-9.

19. Pollack A, Zagars GK, Smith LG, et al. Preliminary results of a randomized radiotherapy dose escalation study comparing 70 Gy to 78 Gy for prostate cancer. J Clin Oncol 2000; 18:3904.

20. Hanks GE, Hanlon AL, Pinover WH, et al. Dose selection for prostate cancer patients based on dose comparison and dose response studies. Int J Radiot Oncol Biol Phys 2000; 46:823e832.

21. Pedro J. Prada M.D, Ph.D1., Juan Cardenal M.D1., Ana García Blanco M.D1., et al. High-dose-rate interstitial brachytherapy as monotherapy in one fraction for the treatment of favorable stage prostate cancer toxicity and long-term biochemical results. Radiotherapy \& Oncology. June 2016, 119(3): 411-416.

22. Donner CS. Pathophysiology and therapy of chronic radiationinduced injury to the colon. Dig Dis 1998; 16:253-261.

23. Wallner K, Roy J, Harrison L. Tumor control and morbidity following transperineal l-125 implantation for stage TI/T2 prostatic carcinoma. J Clin Oncol 1996;14:449-453.

24. Merrick GS, Butler WM, Dorsey MS, et al. Rectal dosimetric analysis following prostate brachytherapy. Int J Radiat Oncol Biol Phys 1999;43:1021- 027.

25. Teshima T, Hanks GE, Hanlon AL, et al. Rectal bleeding after conformal 3D treatment of prostate cancer: Time to occurrence, response to treatment and duration of morbidity. Int J Radiat Oncol Biol Phys 1997;39:77- 83.

\section{Tables}

Due to technical limitations, table 1 is only available as a download in the Supplemental Files section.

\section{Figures}




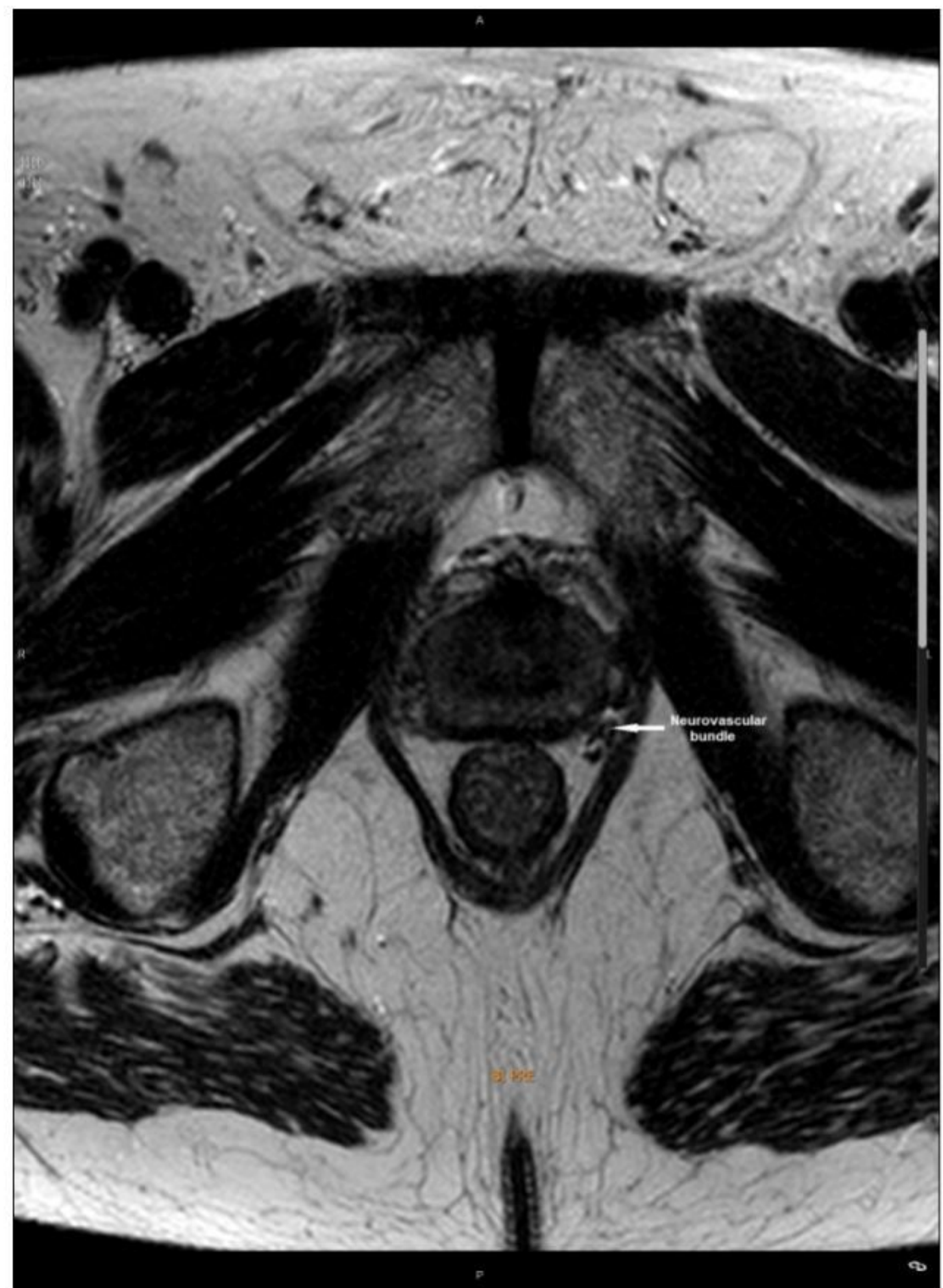

Figure 1

Magnetic resonance image identifying the neurovascular bundle. 


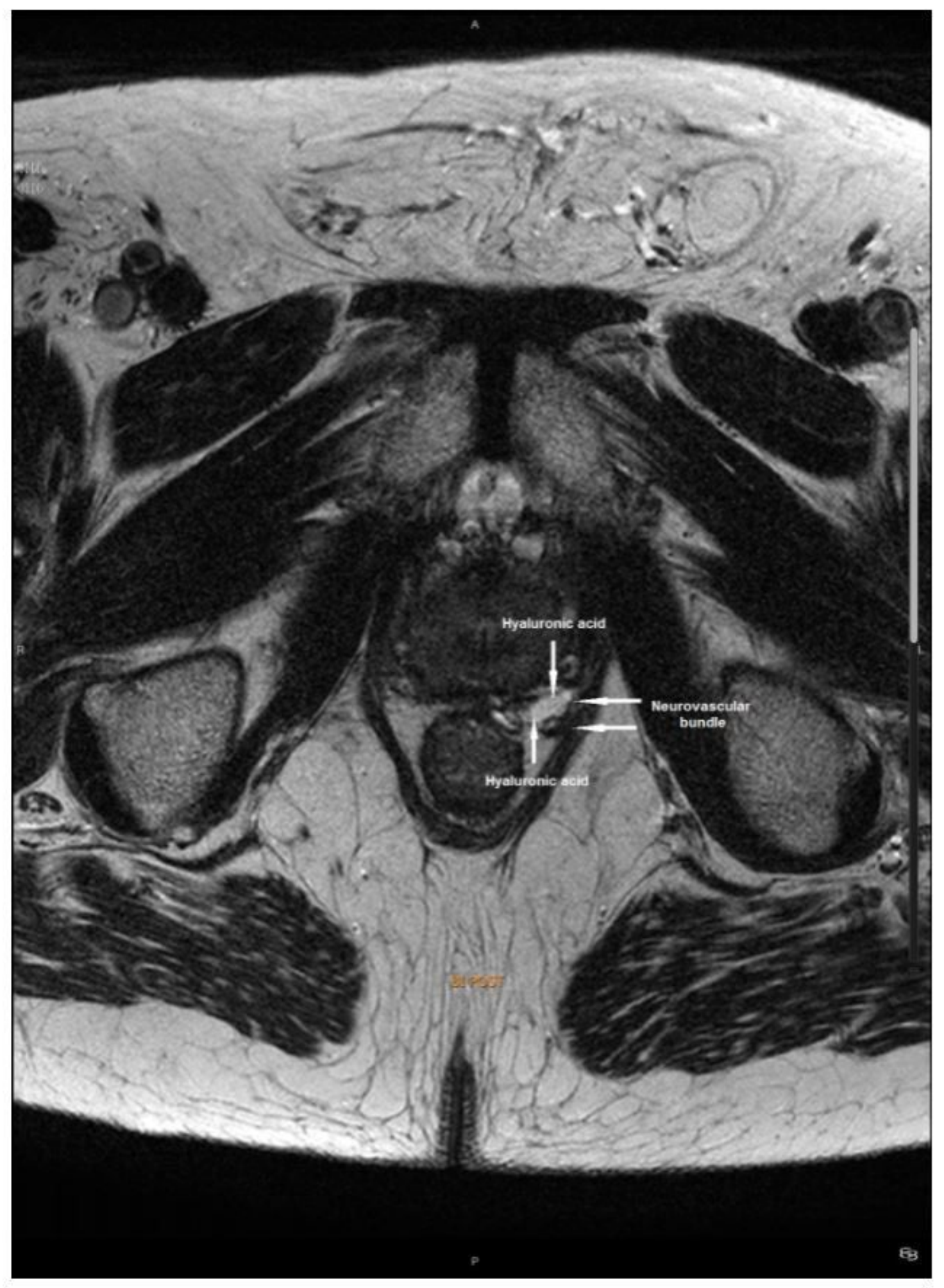

Figure 2

Magnetic resonance image demonstrating the additional space between the prostate capsule and neurovascular bundle.

\section{Supplementary Files}


This is a list of supplementary files associated with this preprint. Click to download.

- Table1.JPG 